\title{
Imaging Blood-Brain Barrier Breakdown Using Multi-Photon Confocal Microscopy
}

\author{
C. J. Guerin ${ }^{1}$, J.D. Cooper ${ }^{2}$, D.A. Pearce ${ }^{3}$ and P. Nicotera ${ }^{1}$ \\ ${ }^{1}$ Medical Research Council Toxicology Unit, Neuroscience Group, Hodgkin Building, Lancaster \\ Road, Leicester, LE1 9HN, UK \\ ${ }^{2}$ Pediatric Storage Disorders Lab, Department of Neuropathology, Box P040, Institute of Psychiatry \\ King's College London, De Crespigny Park, London, SE5 8AF \\ ${ }^{3}$ Center for Aging and Developmental Biology and Department of Biochemistry and Biophysics, \\ University of Rochester School of Medicine and Dentistry, Rochester, NY 14642, USA
}

Breakdown of the bood-brain barrier (BBB) is a feature of many neurodegenerative diseases including multiple sclerosis ${ }^{1}$, AIDS encephalopathy ${ }^{2}$, and cerebral malaria ${ }^{3}$. Successful imaging of this phenomena has been achieved at the light and electron microscopic level using various tracers and methods. However, limitations of the methodology made it difficult to obtain high resolution three dimentional reconstructions of large tissue samples. We have used a combination of injected fluorescent dextrans combined with immunocytochemistry, multi-photon confocal microscopy and $3 \mathrm{D}$ computer reconstructions to image this process in animal model systems.

Blood-brain barrier breakdown was imaged in two model systems. Animals were treated with specific neurotoxicants producing focal lesions or had genetic manipulation which knocked out a gene known to be involved in the juvenile form of Batten's disease. One hour before sacrifice animals were injected with combinations of fluorescently tagged dextrans of differing molecular weights. Tissues were perfusion fixed, embedded in agarose and sectioned into $80-200 \mu \mathrm{m}$ slices on a vibrating microtome. Some animals had further immunocytochemical procedures performed to localize neuronal and glial cells. Sections were inspected on a Zeiss LSM 510 confocal microscope equipped with a Tsuanmi Ti/Sp laser and data sets were reconstructed using Bitplane software running on a Silicon Graphics Octane 2 computer.

Areas of BBB breakdown could be detected by leakage of dextrans through vascular tight junctions into brain tissue. Multi-photon microscopy was able to visualize this pathology throughout thick sections without loss of signal or photobleaching of fluorochromes. Three dimensional reconstructions were able to clearly delineate the extent and molecular weight specificity of the lesions. Simultaneous multiple label immunocytochemistry allowed for visualization of cellular changes associated with the breakdown. ${ }^{4}$

\footnotetext{
${ }^{1}$ Smith KJ, McDonald WI. 1999. The Pathophysiology of multiple sclerosis: the mechanisms underlying the production of symptoms and the natural history of the disease. Philos Trans R Soc Lond Biol Sci 354:1649-1673

${ }^{2}$ Dallasta LM, Pisarov LA, Esplen JE, Werley JV, Moses AV, Nelson JA, Achim CL. 1999. Blood-brain barrier tight junction disruption in human immunodeficency virus-1 enchephalitis. Am J Pathol 155:1915-1927

${ }^{3}$ Brown H, Hien TT, Day N, et al. Evidence of blood-brain barrier dysfunction in human cerebral malaria. Neuropathology and Appl Neurobiol 1999 25:331-340

4 This work has been supported by a grant from the Batten's Disease Support and Research Association and The Medical Research Council of Great Brittan.
} 


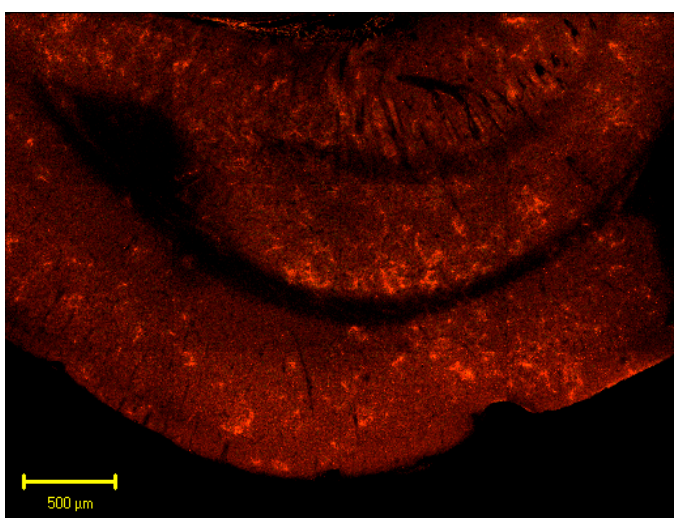

A

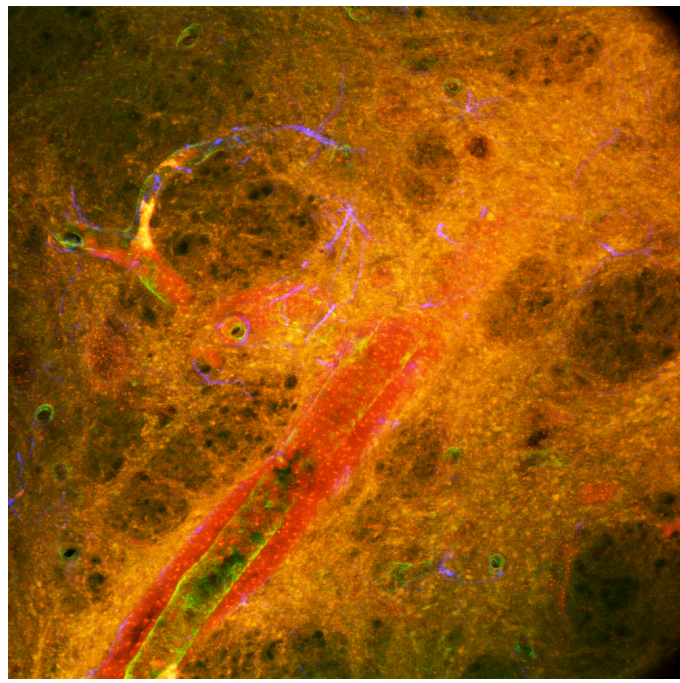

$\mathrm{C}$

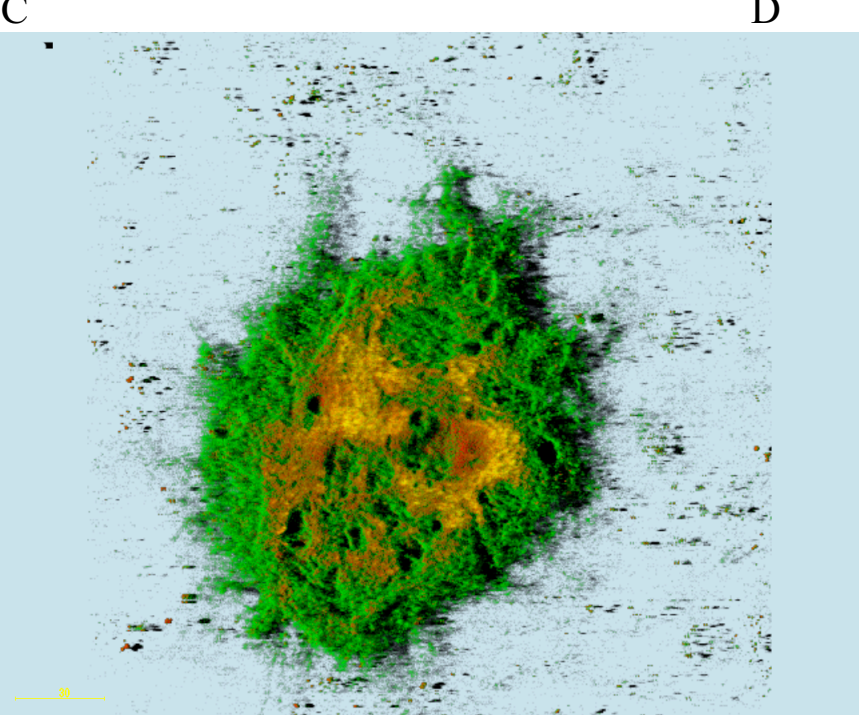

B
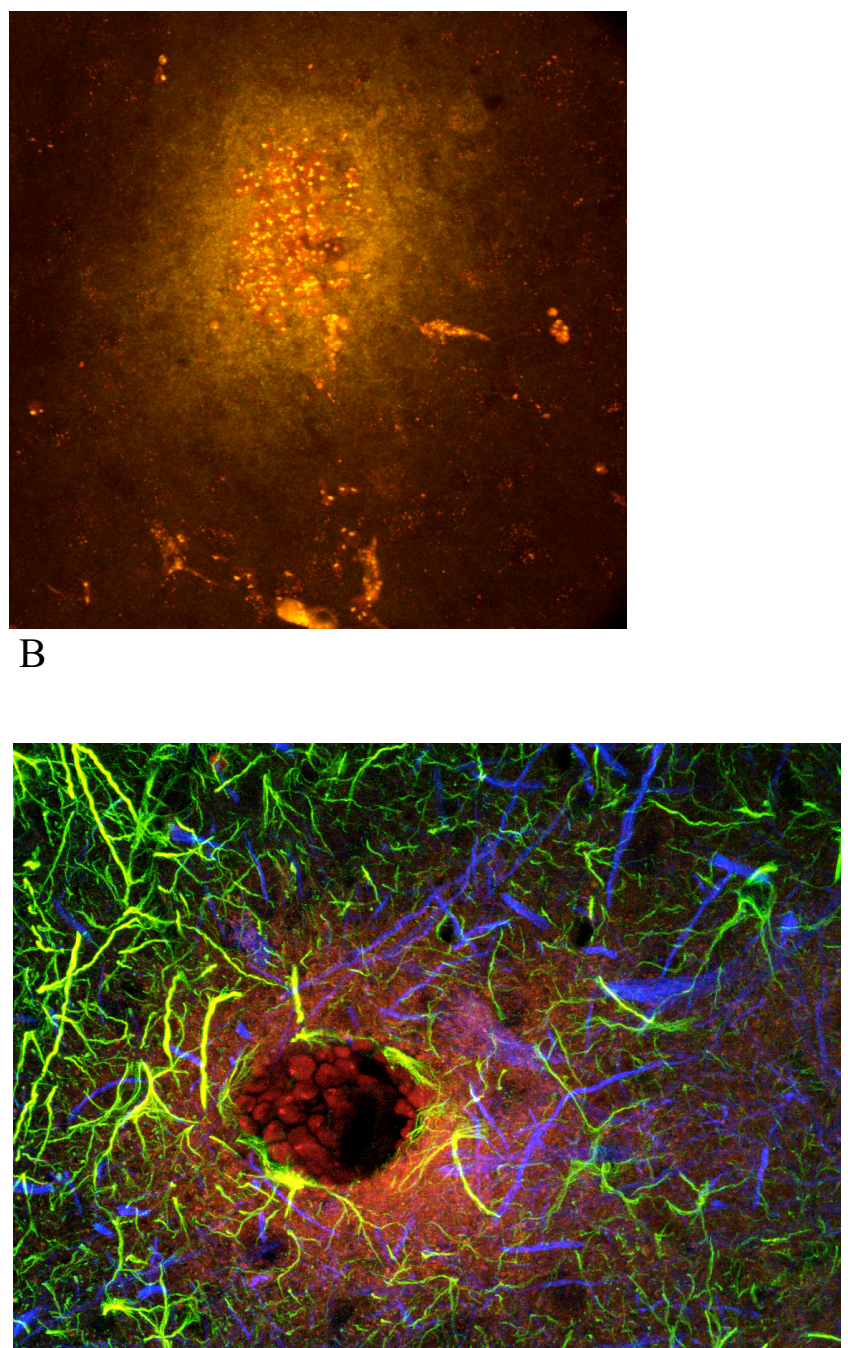

A. Low power high resolution scan of striatum (top), hippocampus and cortex showing multiple areas of BBB breakdown.

B. Higher power micrograph of leaking blood vessel (yellow).

C. Confocal $\mathrm{Z}$ series projection of leaking blood vessel with sleeve hemorrhage (red), labelled glial cells (blue) and vessel walls (green).

D. High power $Z$ series projection of leaking vessel (red) with glia (green) and neurons (blue).

E. 3D volume rendering of dextran 'cloud' surrounding leaking vessel. 